\title{
Correspondence Reduced Density of DISCI Expressing Astrocytes in the Dentate Gyrus but not in the Subventricular Zone in Schizophrenia
}

\author{
\begin{tabular}{l}
\hline Hans-Gert Bernstein*, , Henrik Dobrowolny', Gerburg Keilhoff', Bernhard Bogerts' and Johann Steiner' \\
'Department of Psychiatry, University of Magdeburg, Leipziger Str. 44, Magdeburg D-39 I 20, Germany; ' Institute of Biochemistry and Cell Biology, \\
University of Magdeburg, Leipziger Str. 44, Magdeburg D-39 I20, Germany
\end{tabular}
}

Neuropsychopharmacology (2018) 43, 457-458; doi:I 0.1038/npp.2017.242

There is evidence for impaired adult neurogenesis in the hippocampal subgranular zone of schizophrenia patients (Reif et al, 2006; Allen et al, 2016 and others). Normal and compromized neurogenesis in the adult brain is influenced by a plethora of factors, one of which might by Disruptedin-Schizophrenia 1 (DISC1) (Figure 1) expressed in astrocytes. In elegant experiments, Terrillion et al (2017), (this journal) have recently shown that expression of mutant DISC1 in mouse astrocytes decreased neural progenitor proliferation, produced increased anxiety and impaired hippocampus-dependent learning and memory. Chronic treatment with D-serine restored decreased adult neurogenesis in mutant DISC1 mice. This study prompted us to look for astroglial DISC1 expression in the dentate gyrus and the subventricular zone of individuals with schizophrenia, since despite growing skepticism (reviewed in Mathieson et al, 2012) DISC1 is regarded a risk gene in schizophrenia. We analyzed the density of DISC1-immunoreactive astrocytes in 18 patients with schizophrenia (mean age 54.5 years; nine males, nine females) and 12 matched psychiatric healthy controls (mean age 55.3 years; seven males, five females). Recruitment of cases and tissue handling were as previously described (Bernstein et al, 2016). To immunolocalize DISC1 we used a polyclonal rabbit DISC1 antiserum (Abcam ab 82038, IgG, immunogen affinity purified) at a dilution of $1: 200$. Sections were then incubated with a biotinylated goat anti-rabbit secondary antibody (Amersham) for the streptavidin-biotin technique. The chromogen 3, 3'-diaminobenzidine $(\mathrm{DAB})$ and ammonium nickel sulfate were used to visualize the reaction product. DISC1 immunoreactive astrocytes were counted at 400 fold magnification. We used the optical disector method employing a counting grid as described for glutamine synthetase immunopositive hippocampal astroglia (Bernstein et al, 2013). Normal distribution of demographical data was demonstrated by Kolmogorov-Smirnov tests. A stepwise multiple regression

\footnotetext{
*Correspondence: Dr Hans-Gert Bernstein, Department of Psychiatry, University of Magdeburg, Leipziger Str. 44, D-39120 Magdeburg, Germany Tel: +49 391 6714249, E-mail: Hans-GertBemstein@med.ovgu.de
}

analysis was performed to control for possible influences on cell densities of age, gender, duration of disease, storage delay, whole-brain volume, and medication. Statistical analyses were performed with the SPSS package version 15.0 (Statistical Product and Service Solutions, Chicago, IL, USA). Spearman's correlation coefficients were calculated to determine the influence of variables which could have confounded the outcomes. A Bonferroni correction for multiple comparisons was used. Significance was defined as $P<0.05$. It was revealed that unlike DISC1 immunoreactive oligodendrocytes (Bernstein et al, 2016), DISC1 expressing astrocytes are only few in number.

Compared with controls we found in schizophrenia patients reduced densities for DISC1 astroglia in the left (controls: $236.9 \pm 47.0$ cells $/ \mathrm{mm}^{3}$; schizophrenia: $95.5 \pm 45.3$ cells $/ \mathrm{mm}^{3} ; P=0.001$ ) and right (controls $189.4 \pm 24.9$ cells/ $\mathrm{mm}^{3}$; schizophrenia: $66.8 \pm 20.1$ cells $\left./ \mathrm{mm}^{3} ; P=0.001\right)$ hippocampal dentate gyrus, but not in the left or right subventricular zone (left: controls: $477.2 \pm 122.0$ cells $/ \mathrm{mm}^{3}$; schizophrenia: $434.6 \pm 139.6$ cells $\left./ \mathrm{mm}^{3}\right)$; right: controls $501.0 \pm 166$ cells $/ \mathrm{mm}^{3}$, schizophrenia $466.5 \pm 189.7$ cells/ $\mathrm{mm}^{3}$ ). Significant differences (hippocampus) survived Bonferroni correction (left $P=0.021$; right $P=0.030$ ). Our findings show that in schizophrenia there are fewer DISC1expressing astrocytes in a brain region with established adult neurogenesis (dentate gyrus), but not in the subventricular zone, a region with questionable adult neurogenesis in humans (Comte et al, 2012). Pletnikov's group has shown that D-serine is able to normalize adult neurogenesis and behavioral deficits in DISC1 mutant mice (Terrillion et al, 2017). As astrocytic DISC1 influences D-serine production (Terrillion et al, 2017), a deficit of DISC1-expressing astrocytes in schizophrenia may lead to reduced local levels of D-serine, which may negatively influence neurogenesis in psychiatric patients. This pathophysiologic aspect warrants further attention in future studies. 

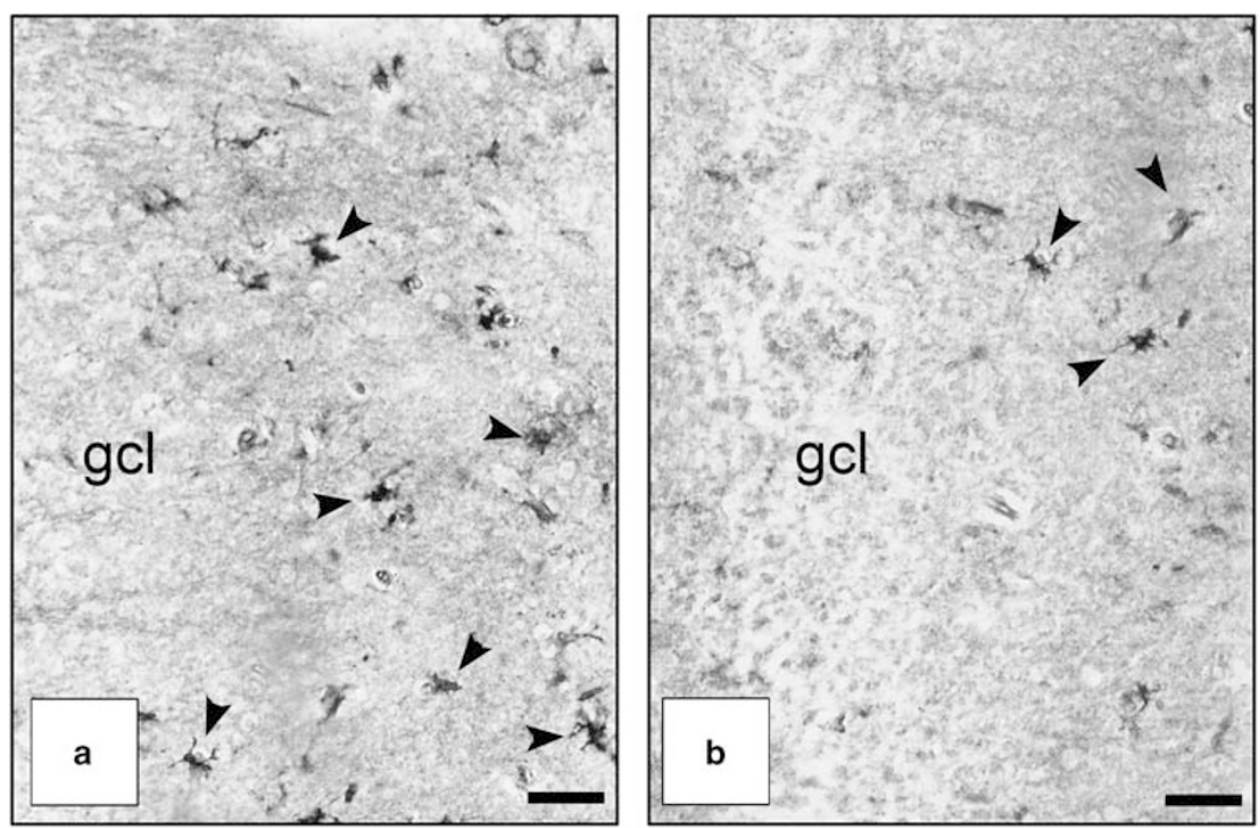

Figure I Immunolocalization of DISCI in human dentate gyrus astrocytes. (a) DISCI immunoreactive astrocytes (arrowheads) in the dentate gyrus of a control case. Granule cell layer (gcl) neurons are only faintly stained. Bar $=30 \mu \mathrm{m}$. (b) Compared with controls there are fewer DISCI immunopositive astrocytes (arrowheads) in the dentate gyrus of schizophrenia patients. Granule cell layer (gcl) neurons are only faintly stained. Bar $=30 \mu \mathrm{m}$.

\section{FUNDING AND DISCLOSURE}

This study was financed by the University of Magdeburg, Germany. The authors declare no conflict of interest.

\section{REFERENCES}

Allen KM, Fung SJ, Weickert CS (2016). Cell proliferation is reduced in the hippocampus in schizophrenia. Aust $N Z J$ Psychiatry 50: 473-480.

Bernstein HG, Tausch A, Wagner R, Steiner J, Seeleke P, Walter M et al (2013). Disruption of glutamate-glutamine-GABA cycle significantly impacts on suicidal behaviour: survey of the literature and own findings on glutamine synthetase. CNS Neurol Disord Drug Targets 12: 900-913.

Bernstein HG, Jauch E, Dobrowolny H, Mawrin C, Steiner J, Bogerts B (2016). Increased density of DISC1-immunoreactive oligodendroglial cells in fronto-parietal white matter of patients with paranoid schizophrenia. Eur Arch Psychiatry Clin Neurosci 266: 495-504.

Comte I, Kotagiri P, Szele FG (2012). Regional differences in human ependymal and subventricular zone cytoarchitecture are unchanged in neuropsychiatric disease. Dev Neurosci 34: 299-309.

Mathieson I, Munafo MR, Flint J (2012). Meta-analysis indicates that common variants at the DISC1 locus are not associated with schizophrenia. Mol Psychiatry 17: 634-641.

Reif A, Fritzen S, Finger M, Strobel A, Lauer M, Schmitt A et al (2006). Neural stem cell proliferation is decreased in schizophrenia, but not in depression. Mol Psychiatry 11: 514-522.

Terrillion CE, Abazyan B, Yang Z, Crawford J, Shevelkin AV, Jouroukhin Y et al (2017). DISC1 in astrocytes influences adult neurogenesis and hippocampus-dependent behaviors in mice. Neuropsychopharmacology 42: 2242-2251. 